\title{
Acute Lobar Nephronia Secondary to Obstruction Due to Urolithiasis*
}

\author{
illke Beyitler' (D), Murat Kocaoğlu² (D), Salih Kavukçu' (D) \\ 'Department of Pediatrics, Near East University Faculty of Medicine, Nicosia, North Cyprus \\ ${ }^{2}$ Department of Radiology, Near East University Faculty of Medicine, Nicosia, North Cyprus \\ ORCID iDs of the authors: I.B. 0000-0002-7758-90I5; M.K. 0000-0002-4674-8634; S.K. 0000-0002-9959-88IX.
}

Cite this article as: Beyitler i, Kocaoğlu M, Kavukçu S. Acute Lobar Nephronia Secondary to Obstruction Due to Urolithiasis. Cyprus J Med Sci. 2020; 6(3): 267-269.

\begin{abstract}
Acute lobar nephronia $(A L N)$ is a focal nonliquefactive bacterial infection affecting one or more renal lobules. Although it is a rare form of urinary infection, it has been diagnosed with increasing rates by the utility of ultrasonography (USG). The inflammatory markers are high in ALN, and it requires longer duration of antibiotic treatment. Effective treatment can prevent its progression to renal abscess. ALN may be seen in healthy children and those with urinary tract abnormalities as well. Here, we presented an infant with ureterolithiasis that developed ALN with prolonged fever. He was treated with intravenous antibiotics, and fever with USG findings were resolved. As ALN produces a mass lesion that may mimic other entities such as abscess or tumors, diagnosis is important for sufficient antibiotic treatment and avoidance of unnecessary invasive operations.
\end{abstract}

Keywords: Lobar nephronia, ureterolithiasis, bacterial infection, focal nephritis

\section{INTRODUCTION}

Acute lobar nephronia (ALN), also known as acute focal bacterial nephritis, is a rare process of bacterial urinary tract infection (UTI), presenting as an inflammation that affects one or more renal lobules. It represents the progressed form of acute pyelonephritis (APN) and the early stage of a developing renal abscess. Clinical presentation involves fever, vomiting, and abdominal pain. Patients have leukocytosis, elevation of C-reactive protein (CRP), pyuria, and infectious agent that may or may not be detected with urine culture. The cause is an ascending infection from the lower urinary tract or by hematogenous spread. The febrile period after antibiotic treatment is longer, and vomiting is more frequent in ALN than in APN. Also, the neutrophil count and CRP levels are higher in ALN. Urinary tract ultrasonography (USG) is very effective in diagnosis describing a hypoechogenic/hyperechogenic and hypoperfused lesion. It may be misinterpreted as an abscess or tumor. ${ }^{1-3}$

\section{CASE PRESENTATION}

A I3-month-old male patient was admitted with fever that he had for 3 days and vomiting for 2 days. Before admission to our hospital, a single dose of intramuscular antibiotic was administered at a different medical center. On admission to our hospital, he had fever as $39-40^{\circ} \mathrm{C}$ and pale skin on physical examination. He had a normal growth and a BCG skar. Laboratory test results included leucocyte $20,580 \mathrm{~mm}^{-3}$, neutrophil $68 \%$, hemoglobin $9.1 \mathrm{~g} \mathrm{dL}^{-1}$, mean corpuscular volume (MCV) $74.8 \mathrm{fL}$, red cell distribution width (RDW) I3, platelets $236,000 \mathrm{~mm}^{-3}, \mathrm{CRP} / 8 \mathrm{mg} \mathrm{dL}^{-1}\left(\mathrm{~N}: 0-0.5 \mathrm{mg} \mathrm{dL}^{-1}\right)$, urine analysis: leucocyte esterase: +++ , pyuria, and bacteriuria.

USG showed a $5 \mathrm{~mm}$ calculus at the distal end of the left ureter (Figure la) and a hyperechogenic, hypoperfused area with a $30 \mathrm{~mm}$ diameter, leading to contour lobulation at the upper pole of the left kidney (Figure lb). Immunoglobulin $\mathrm{G}$, $A$, and $M$ levels were normal according to his age.

The patient was given ceftriaxone treatment, and fever stopped on the third day. Urine culture was sterile but pyuria continued. Acidoresistant bacteria was negative in urine culture. Amikacin was added to the treatment and given for 7 days. Ceftriaxone was planned to be given for 14 days. 


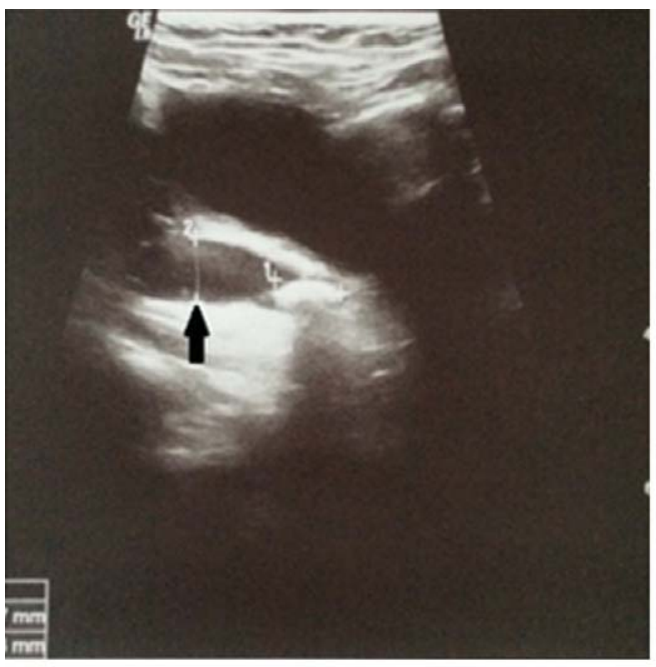

(a)

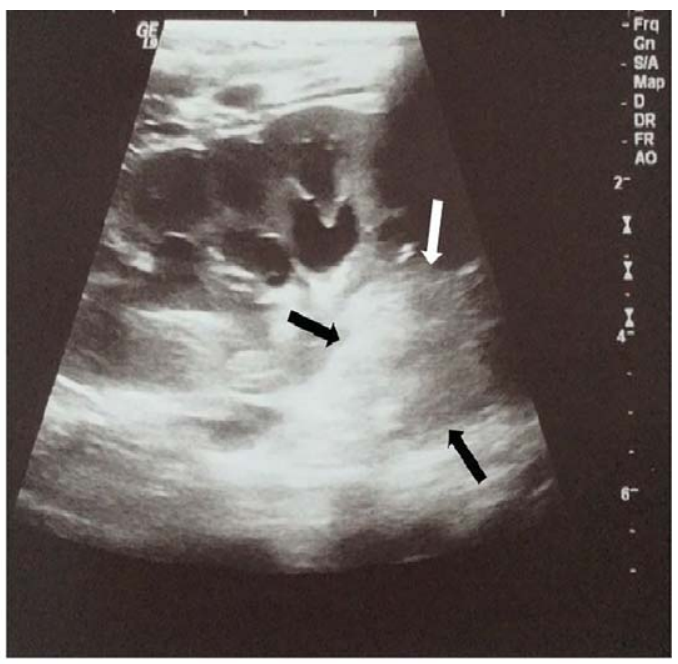

(b)

Figure I. a,b. (a) Parasagittal sonography through the urinary bladder reveals an ureteral stone just before the ureterovesical junction (arrow) and proximal ureteral dilatation. (b) Coronal ultrasonography shows a well-defined cortical hyperechogenic area at the upper pole of the left kidney (arrows). Collecting system is also dilated

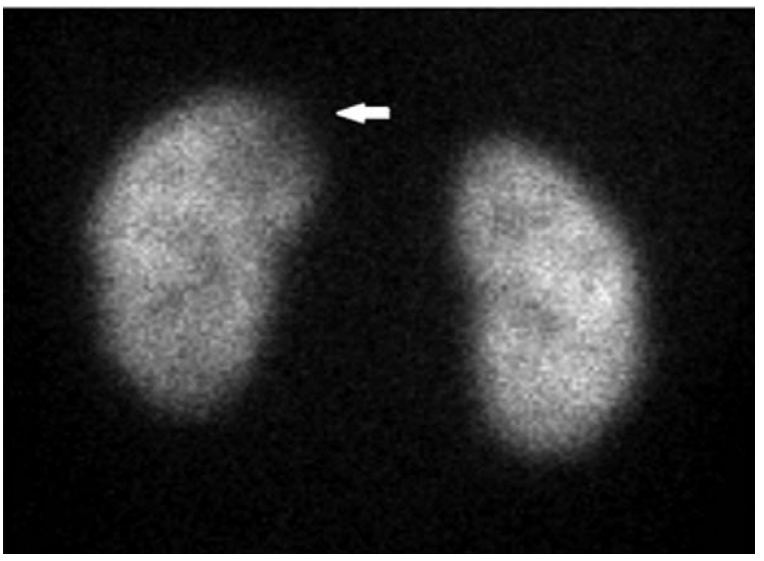

Figure 2. Paranchymal dysfunction at the upper pole of the left kidney (arrow)

Abdominal USG on the fifth day of hospitalization showed increased left pelvicaliectasia and dilatation along the whole ureter with a $8.5 \mathrm{~mm}$ diameter at the distal end. Calculus was measured $9 \mathrm{~mm}$ at the distal end of the ureter, and this measurement was more accurate due to ureteral dilatation. Focal

\section{Main Points}

- ALN is a severe inflammatory renal bacterial infection that requires a long duration of antibiotic treatment.

- It can both occur in children with a urinary system abnormality or in those with normal anatomy.

- ALN is associated with high incidence of renal scar formation. Diagnosis and treatment are essential to avoid complications and unnecessary interventions. area at the upper pole of the left kidney disappeared on this imaging. CRP was $4.8 \mathrm{mg} \mathrm{dL}^{-1}$, pyuria continued, and repeated urine culture was again normal.

The patient was directed to pediatric surgery after the seventh day of admission. Pyuria was resolved, and CRP was $2.3 \mathrm{mg} \mathrm{dL}^{-1}$. Detailed tests for the type of ureterolithiasis showed hyperoxaluria. Urinary oxalate/creatinine ratio was 0.08 (normal: <0.05). Calculus was removed ureteroscopically but could not be sent to stone analysis because it totally split up during the operation. Antibiotic treatment completed to 14 days. The presented case did not have vesicoureteral reflux (VUR), but he had renal scar at upper pole of the left kidney on scintigraphy (Figure 2). Nitrofurantoin prophylaxis was initiated after treatment.

\section{DISCUSSION}

A high incidence of urinary tract anomalies (22\%) is found to be related to ALN. VUR was the most frequently (17\%) seen anomaly, followed by hydronephrosis (7\%). Less common pathologies are small kidney, single kidney, hydroureter, urethra with double orifice, and Cobb's collar. ${ }^{4}$

In another retrospective investigation of 25 pediatric ALN cases, 12 had urinary tract abnormalities (eight $\vee U R$, one megaureter, one urethral valve, one unilateral renal hypoplasia, and one megacystis megaureter with renal dysplasia). Twenty of them resolved with antibiotics, whereas renal parenchymal cysts remained in three cases, and focal scar in two cases. ${ }^{5}$

Sixteen children with ALN were retrospectively reviewed, and all of them had a predisposing condition. Three of them had VUR not requiring surgery, one had multiple renal calyceal diverticula, one had leukemia, and one had Hinman syndrome. Ten children needed surgery: four high grade VUR, one bladder diverticulum, two renal and perirectal abscess, one renal mass (Wilms tumor), one ureteric calculus, and one bladder calculus. The patient who needed stent for ureteric calculus was treated successfully with antibiotics, and ALN disappeared as in the other patients. ${ }^{6}$ 
Lobar nephronia in our patient was a consequence of obstruction and infection due to ureterolithiasis. Ureterolithiasis occured because of hyperoxaluria. ALN occurring directly due to hyperoxaluria was not defined in literature. ALN in this case resulted from an UTI due to the obstruction by any type of calculus.

In febrile UTIs, the diagnosis of ALN is possible with urinary USG performed by an expert, which is a very good and effective screening method. Computed tomography is described as a more sensitive and specific imaging method but has disadvantages like radiation and being invasive. ${ }^{7}$

In the beginning, the patient was considered as APN secondary to obstruction due to ureterolithiasis according to clinical signs and treated as APN. Sterility of urine culture was attributed to antibiotic treatment before admission. However, insufficient antibiotic response and definition of an inflammatory area with USG changed our diagnosis from APN to ALN.

ALN was defined by USG, and inflammation area regressed in I week. Voiding cystourethrogram was normal, but hypoactive lesion persisted as a scar on scintigraphy. In other words, in patient with ALN diagnosis, clinical condition, acute phase reactants, and acute inflammation on USG recovered I week after ceftriaxone and amikacin combination. However, scintigraphic signs persisted as scar. Antibiotics and removal of obstruction did not prevent scar formation. Nitrofurantoin was initiated after considering patient's age, history of UTI, and renal scar. Relapse of UTI or calculus was not observed during the I year follow-up.

ALN is a severe infection requiring long antibiotic duration, in which the patients should be treated with intravenous antibiotics for 2-3 weeks. It results in a high incidence of renal scarring diagnosed on follow-up with renal cortical scintigraphy. Frequency of renal scar development is not always dependent on treatment duration. In a prospective study with 109 ALN and 109 APN pediatric patients, renal scar incidence was similar in both groups taking antibiotic for 2 and 3 weeks. However, renal scar was much higher in ALN (89\%) than in APN (34.9\%) patients. Children with longer duration of fever and higher inflammation markers were also more susceptible for scar. ${ }^{8}$

The presented case is a rare condition as an ureterolithiasis predisposing ALN that was treated with intravenous antibiotics and evaluated with renal cortical scintigraphy. Calculus analysis revealed hyperoxaluria. Hyperoxaluria, in this case, may directly cause renal damage as defined in the literature. Other than crystalluria, genetical polymorphisms in inflammasome-dependent innate immunity and toll-like receptors may be responsible for ALN development. ${ }^{9-11}$
As ALN produces a mass lesion that may mimic other entities, diagnosis is important for sufficient antibiotic treatment and avoidance of unnecessary invasive operations.

Informed Consent: Verbal informed consent was obtained from all participants who participated in this study.

Peer-review: Externally peer-reviewed.

Author Contributions: Conception - I.B., S.K; Design - I.B., S.K.; Supervision - I.B., M.K., S.K.; Data Collection and/or Processing - I.B.; Analysis and Interpretation: I.B., M.K., S.K.; Literature Review - I.B., S.K.; Writing I.B., S.K.; Critical Review - I.B., M.K., S.K.

Conflict of Interest: The authors have no conflicts of interest to declare.

Financial Disclosure: The authors declared that this study has received no financial support.

\section{REFERENCES}

I. Sheu J. Acute lobar nephronia in children. Pediatr Neonatol. 2015;56(3):|4|-|42. [CrossRef]

2. Chen WL, Huang IF, Wang JL, et al. Comparison of acute lobar nephronia and acute pyelonephritis in children: A single-center clinical analysis in Southern Taiwan. Pediatr Neonatol. 2015;56(3):176-182. [CrossRef]

3. Alaygut D, Bayram M, Soylu A, Turkmen M, Kavukcu S. Acute focal bacterial nephritis developed in a healthy child. Turk $J$ Pediatr. 2013;55(2):226-228.

4. Yang CC, Shao PL, Lu CY, et al. Comparison of acute lobar nephronia and uncomplicated urinary tract infection in children. J Microbiol Immunol Infect. 2010;43(3):207-214. [CrossRef]

5. Seidel T, Kuwertz E, Kaczmarek $S$, et al. Acute focal bacterial nephritis in 25 children. Pediatr Nephrol. 2007;22(II):1897-190I. [CrossRef]

6. Uehling DT, Hahnfeld LE, Scanlan KA. Urinary tract abnormalities in children with acute focal bacterial nephritis. BJU Int. 2001;85(7):885-888. [CrossRef]

7. Rianthavorn P. Progression and resolution of acute focal bacterial nephritis. Iran J Kidney Dis. 20Il;5(4):27I-274.

8. Cheng $\mathrm{CH}$, Tsau $\mathrm{YK}$, Chang $\mathrm{CJ}$, et al. Acute lobar nephronia is associated with a high incidence of renal scarring in childhood urinary tract infections. Pediatr Infect Dis J. 2010;29(7):624-628. [CrossRef]

9. Darisipudi MN, Knauf F. An update on the role of the inflammasomes in the pathogenesis of kidney disease. Pediatr Nephrol. 2016;3|(4):535-544. [CrossRef]

10. Cheng CH, Lee YS, Chang CJ, Lin JC, Lin TY. Genetic polymorphisms in inflammasome-Dependent innate immunity among pediatric patients with sever renal parenchymal infections. PloS One. 2015;I0(10):e0I40I28. [CrossRef]

II. Cheng CH, Lee YS, Chang CJ, Lin TY. Genetic polymorphisms in toll-like receptors among pediatric patients with renal parenchymal infections of different clinical severities. PloS One. 2013;8(3):e58687. [CrossRef] 